\title{
Ab initio Investigation of the Structure and Electronic Properties of Normal Spinel $\mathrm{Fe}_{2} \mathrm{SiO}_{4}$
}

\author{
Idris Muhammad Chiromawa ${ }^{a, b}$, Amiruddin Shaaria,*, Razif Razalia, \\ Summanuwa Timothy Ahams ${ }^{a, c}$, Mikailu Abdullahi ${ }^{b}$
}

aphysics Department, Universiti Teknologi Malaysia, 81310 Skudai Johor, Malaysia; 'b Physics Department, Sule Lamido University Kafin-Hausa, Jigawa State, Nigeria; ${ }^{~ P h y s i c s ~ D e p a r t m e n t, ~ A d a m a w a ~ S t a t e ~ U n i v e r s i t y ~ M u b i, ~ N i g e r i a ~}$

Abstract Transition metal spinel oxides have recently been predicted to create efficient transparent conducting oxides for optoelectronic devices. These compounds can be easily tuned by doping or defect to adapt their electronic or magnetic properties. However, their cation distribution is very complex and band structures are still subject to controversy. We propose a complete density functional theory investigation of fayalite $\left(\mathrm{Fe}_{2} \mathrm{SiO}_{4}\right)$ spinel, using Generalized Gradient Approximation (GGA) and Local Density Approximation (LDA) in order to explain the electronic and structural properties of this material. A detailed study of their crystal structure and electronic structure is given and compared with experimental data. The lattice parameters calculated are in agreement with the lattice obtained experimentally. The band structure of $\mathrm{Fe}_{2} \mathrm{SiO}_{4}$ spinel without Coulomb parameter $U$ shows that the bands close to Fermi energy appear to be a band metal, with four iron $d$-bands crossing the Fermi level, in spite of the fact that from the experiment it is found to be an insulator.

Keywords: structural properties, transparent conducting oxides, DFT, Coulomb parameter (U)

\section{Introduction}

Transparent conducting oxides (TCOs) are electrically conductive materials with comparably low absorption of electromagnetic waves within the visible region of the spectrum. They belong to an exceptional family of oxides, possessing two antagonistic physical properties, high optical transparency to visible light and high electrical conductivity carrier concentrations [1]. Due to these properties, the TCOs are technologically classified as an important class of materials in the field of optoelectronics $[2,3]$. TCOs have found a wide variety of technological applications, such as solar cells, low emissivity windows, window defrosters, flat-panel displays, blue or ultraviolet light-emitting diodes (LEDs), liquid crystal displays, dimming rear-view mirrors, semiconductor lasers, energy-conserving touch screens, light-emitting displays, invisible and security circuits [4,5]. Also, some new applications of TCOs have been suggested recently such as holographic recording media, write-once read-many-times memory chips (WORM), electronic ink etc. [1,6]. Most of the TCOs have n-type conductivity and the development of efficient p-type TCOs is one of the key goals of researchers. High conductivity p-type TCOs similar to the high-performance n-type TCOs would be a major breakthrough, facilitating advanced devices and applications [7]. Spinel structures have the common formula of $\mathrm{TB}_{2} \mathrm{X}_{4}$, where $\mathrm{X}$ can be oxygen (oxides) or a chalcogens element, such as sulfur (thio-spinels) and selenium (selenospinels) while $\mathrm{T}$ and $\mathrm{B}$ can be divalent, trivalent, or tetravalent cations. Spinel oxides have been recognized as promising p-type TCO semiconductors, which can be a substitute to the n-type indium doped tin- oxide (ITO) [8]. The perspectives of usage of the spinel oxides as p-type transparent 
conducting oxide semiconductors and for other applications have stimulated widespread experimental and theoretical studies on this exciting class of materials [9-11]. Spinel oxides are categorized by their robust properties, such as high strength, good electrical conductivity, high resistance to chemical attack, high melting temperature and large fundamental bandgap [10,11]. Fayalite $\left(\mathrm{Fe}_{2} \mathrm{SiO}_{4}\right)$ is one of the promising transparent semiconducting for various technological applications. The spinel oxide $\mathrm{Fe}_{2} \mathrm{SiO}_{4}$ was synthesized and its crystalline structure was identified a long time ago [12]. Some of the fundamental physical properties of $\mathrm{Fe}_{2} \mathrm{SiO}_{4}$, such as structural and electronic properties, have been already investigated experimentally and theoretically by first-principles calculations [13-16]. However, the theoretical band gap value of $\mathrm{Fe}_{2} \mathrm{SiO}_{4}$ is still under debate.

Fortuitously, there are many first-principles techniques that can be used to describe accurately the electronic structure of semiconductors and insulators [17-20]]. For instance, the recently proposed modified Becke-Johnson (MBJ) potential and thereafter the well-known GW approximation, but the major problem with these techniques is they are too expensive. However, there are other first-principles methods (GGA and LDA) that are cheaper and less time-consuming. Both the GGA and LDA involve the use of pseudopotentials (PPs). Here, we apply the latter methods to study systematically the structure and electronic band parameters of spinel $\mathrm{Fe}_{2} \mathrm{SiO}_{4}$ successively. In addition, we also focused not only on predicting the real value of the fundamental band gap in this material but also on other key properties in TCOs, like the second bandgap (between the two lowest conduction bands) [21]]. The results will resolve the discrepancies where exist on this spinel material and also indicate its suitability for optical devices.

\section{Materials and methods}

\section{Computational details}

In this work, the electrical properties of the $\mathrm{Fe}_{2} \mathrm{SiO}_{4}$ were investigated using density functional theory (DFT) with the pseudopotential plane-wave method as implemented in Quantum Espresso (QE) package [22]. The electronic calculation was performed using the DFT-GGA/LDA and GGA+U/LDA+U. All calculations were spin-unpolarized and the exchange-correlation terms were described using the Perdew-Burke-Ernzerhof (PBE) and Perdew-Zunger (PZ) functional. For the DFT- GGA $(G G A+U)$ calculation, the norm-conserving pseudopotentials including the $\mathrm{Fe}(3 \mathrm{~s}, 4 \mathrm{~s}, 3 \mathrm{p}, 3 \mathrm{~d}), \mathrm{Si}(3 \mathrm{~s}, 3 \mathrm{p})$ and $\mathrm{O}(2 \mathrm{~s}$, $2 p)$ state in its valence shell were used [23]]. The wave functions were expanded in-plane waves up to the kinetic energy cutoff of $350 \mathrm{Ry}$ and convergence criteria for the energy of $10^{-8} \mathrm{eV}$ were chosen while for the DFT-LDA (LDA+U) the $\mathrm{Fe}(3 \mathrm{~s}, 4 \mathrm{~s}, 3 p, 3 \mathrm{~d})$, $\mathrm{Si}(3 \mathrm{~s}, 3 p)$ and $\mathrm{O}(2 \mathrm{~s}, 2 \mathrm{p})$ state were used. A planewave kinetic energy cut-off of $450 \mathrm{Ry}$ was selected [24]]. These ensure a good convergence of the computed lattice constant (see Table 1). In order to take into account the on-site Coulomb interactions between $3 d$ electrons, the values of the Coulomb integral $U=6.8 \mathrm{eV}$ and Hund's exchange $J=0.89$ $\mathrm{eV}$ (from Ref. [25]) for $\mathrm{Fe}^{2+}$ in $\mathrm{Fe}_{2} \mathrm{SiO}_{4}$ in the $\mathrm{GGA}+U$ and $\mathrm{LDA}+U$ calculations were used. We performed our calculations on the 56 -atoms unit cell of the $\mathrm{Fe}_{2} \mathrm{SiO}_{4}$ spinel structure. An $8 \times 8 \times 8$ Monkhorst-Pack k-point mesh was used for the cubic structure used to obtain a well-converged sampling of the Brillouin zone.

\section{Results and discussion}

\section{Crystal Structural}

The spinel $\mathrm{Fe}_{2} \mathrm{SiO}_{4}$ fayalite belongs to $\mathrm{Fd}-3 \mathrm{~m}$ (227) space group symmetry and it crystallizes in an fcc (face-centered cubic) lattice. A unit cell of $\mathrm{Fe}_{2} \mathrm{SiO}_{4}$ is shown in Figure 1. There are 56 atoms in the unit cell or eight unit formulas $\left(8 \mathrm{Fe}_{2} \mathrm{SiO}_{4}\right)$. It contains $8 \mathrm{Si}$ cations, $16 \mathrm{Fe}$ cations and $32 \mathrm{O}$ anions in the unit cell, which are located at the Wyckoff positions: $8 a\left(\frac{1}{8}, \frac{1}{8}, \frac{1}{8}\right)$, tetrahedra sites, $16 d\left(\frac{1}{2}, \frac{1}{2}, \frac{1}{2}\right)$, octahedral sites, and $32 e(u, u, u)$, respectively. Every atom of $\mathrm{Si}$ is surrounded by $4 \mathrm{O}$ atoms, forming a regular tetrahedron and every atom of $\mathrm{Fe}$ is surrounded by $6 \mathrm{O}$ atoms, forming a regular octahedron. The FeO6 octahedron and $\mathrm{SiO} 4$ tetrahedron are connected to each other through common edges.

At the initial step of our calculation, full structural optimization and convergence test of the considered material was performed to determine the equilibrium structural parameters, including the lattice parameter (a). To do that, total energy was calculated for a series of primitive cell volumes, where the atomic positions were allowed to relax for each volume. The resulting total energy-volume curve was 
fitted to the Birch-Murnaghan equation of state [26] to determine the equilibrium primitive cell volume, bulk modulus $\boldsymbol{B}$ and its pressure derivative $\mathbf{B}^{\prime}$. The optimized structural parameters using the GGA, $G G A+U$, LDA and $L D A+U$ are displayed in Table 1 together with the existing experimental and theoretical data. One can appreciate from the Table 1 that, there is an excellent agreement between the GGA+U optimized lattice parameter value and the corresponding experimental one. The relative deviation $(\boldsymbol{d})$ of the calculated lattice parameter $\left(\boldsymbol{a}_{\text {calculated }}\right)$ from the experimental $\left(\boldsymbol{a}_{\text {exprimental }}\right)$ one which is defined as:

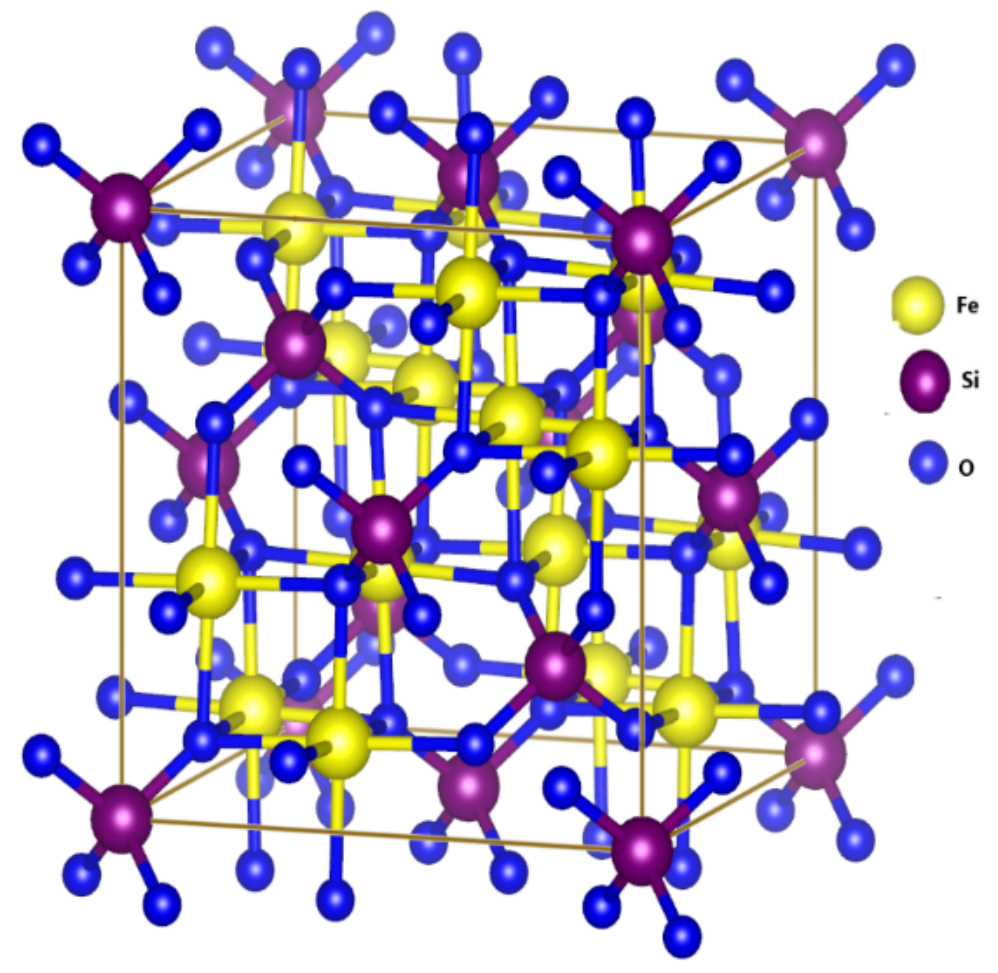

Figure 1. The $\mathrm{Fe}_{2} \mathrm{SiO}_{4}$ fayalite Crystal Structure: the octahedron Fe sites are represented by yellow balls showing $6 \mathrm{O}$ atoms surround every Fe atom and $4 \mathrm{O}$ atoms, forming a regular tetrahedron, surround every Si atom.

$\boldsymbol{d}=\left(\frac{\boldsymbol{a}_{\text {calculated }-a_{\text {experimental }}}}{\boldsymbol{a}_{\text {experimental }}}\right) \times(\mathbf{1 0 0} \%)$ is less than $-1.07 \%$ for GGA and $-0.27 \%$ for LDA while that of GGA $+U$ is $-0.97 \%$ and $-0.12 \%$ in the case of LDA+U. It is significant that the GGA/LDA and $\mathrm{GGA}+U / \mathrm{LDA}+U$ methods both accurately predict the right shape of the unit cell. All investigated properties were calculated at the optimized structural parameters obtained in this work.

Table 1. Optimized equilibrium lattice parameter (a, in $\AA$ ) for $\mathrm{Fe}_{2} \mathrm{SiO}_{4}$ fayalite together with the available experimental result in the literature.

\begin{tabular}{ccc}
\hline Method & al $\AA$ & VI $\AA^{3}$ \\
\hline GGA & 8.146 & 540.547 \\
LDA & 8.256 & 562.742 \\
GGA+U & 8.154 & 542.141 \\
LDA+U & 8.244 & 560.291 \\
Experiment [25] & 8.234 & 558.255 \\
\hline
\end{tabular}




\section{Electronic properties}

The use of electronic structure methods, adept for correctly calculating the bandgap for optical transparency is useful in finding new or improved optoelectronics materials. The calculated electronic energy bands dispersions for the optimized crystal structure of the considered material along the particular high-symmetry points within the Brillion zone (BZ) via the DFT and DFT $U$ are illustrated in Figure 2. For both GGA-PBE and LDA-PZ the electronic band gap entirely disappears (see figure 2). This is due to the well-known band gap underestimation caused by the incorrect treatment of electron exchange in DFT[27,28]. As a result, the Fermi level falls within some long, extended bands, and the material appears to be metallic. Thus, DFT is insufficient for accurately predicting the electronic properties of the ternary oxide under study, and is not appropriate for screening this material. It can be observed from figure 3 that, the studied material using both $G G A+U$ and LDA+U is a direct band gap semiconductor, where both the VBM (valence band maximum) and CBM (conduction band minimum) are located at the point $W$ in the BZ. The main features (band dispersions) of the GGA $U$ and $L D A+U$ band structures are basically identical, except that the $G G A+U$ band gap is much higher than the $L D A+U$ one. The $G G A+U$ band gap is $3.11 \mathrm{eV}$ while $L D A+U$ is $2.88 \mathrm{eV}$. The value of the experimental band gap of the studied material was reported to be $4.2 \mathrm{eV}$ obtained using spectroscopic technique.

Thus, one can state that $G G A+U$ provides a reasonable simple method that can be a viable alternative to the computationally expensive approaches for the calculation of band gaps of strongly correlated materials with an acceptable accuracy. It is worthy to note that the discrepancy between. experimental data reported by different researchers for the $\mathrm{Fe}_{2} \mathrm{SiO}_{4}$ band gap is probably due to the experimental errors that usually arises from the used measurement technique and sample quality. In addition, this inconsistency between experimental data can be explained by the fact that the band gap value depends on the temperature at which the measurement is done. Also it might be worthy revealing that, the minor differences between our GGA+U and $L D A+U$ band gaps and the earlier reported results using other techniques could be attributed to the fact that these values are derived from calculations performed at slightly different values of the optimized structural parameters; the band gap value is sensitive to the structural parameter values.

(a)

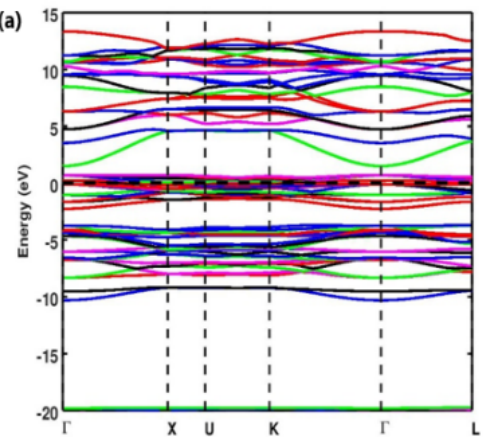

(c)

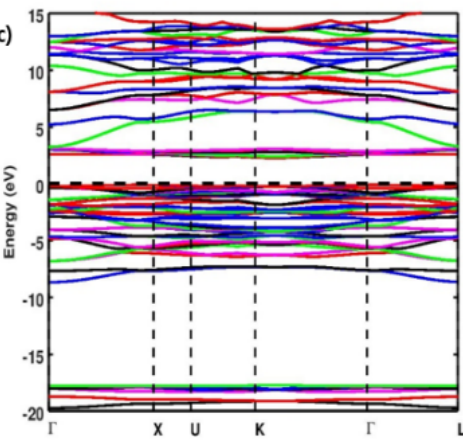

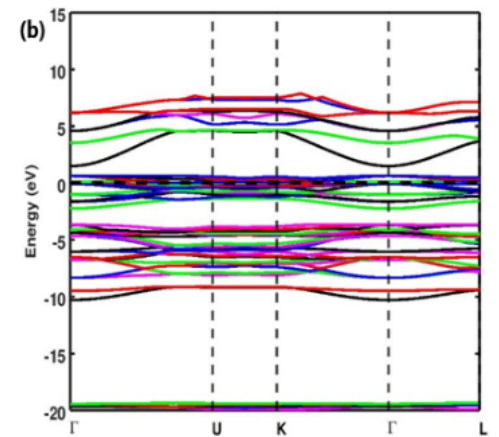

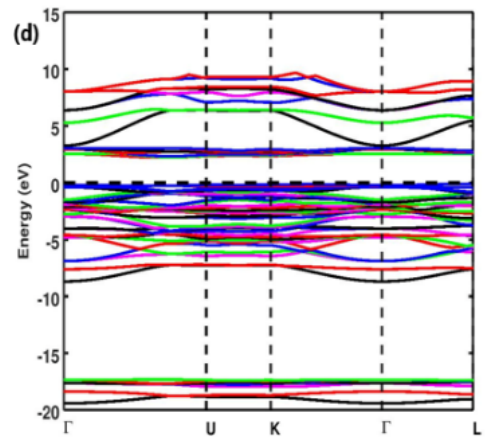

Figure 2. Band structure of $\mathrm{Fe}_{2} \mathrm{SiO}_{4}$ using (a) GGA-PBE, (b) LDA-PZ, (c) GGA+U/PBE+U, (d) $L D A+U / P Z+U$ method. 
From the study of the energy band dispersions at the energy band extremes, qualitative data can be obtained about the ability of the studied material to easily conduct electricity. Figure 2 shows that the valence energy bands around the VBM are more dispersive than the conduction bands around the CBM, this indicate that the effective mass of the electron will be heavier than that of the hole [29]. This result suggests that the $\mathrm{n}$-doped $\mathrm{Fe}_{2} \mathrm{SiO}_{4}$ should be more advantageous for optoelectronic devices performance than the $p$-doped ones, while the electrical conductivity by valence band electrons should be more promising than that by conduction band holes.

Table 2. Calculated fundamental band gap $\left(E_{g}\right)$ for $\mathrm{Fe}_{2} \mathrm{SiO}_{4}$ fayalite together with the existing theoretical and experimental findings.

\begin{tabular}{cc}
\hline Method & $E_{g} / \mathrm{eV}$ \\
\hline This work GGA+U & 3.11 \\
LDA+U & 2.88 \\
Experiment & $4.22[30]$ \\
Others & $2.21[13], 2.52[16]$ \\
\hline
\end{tabular}
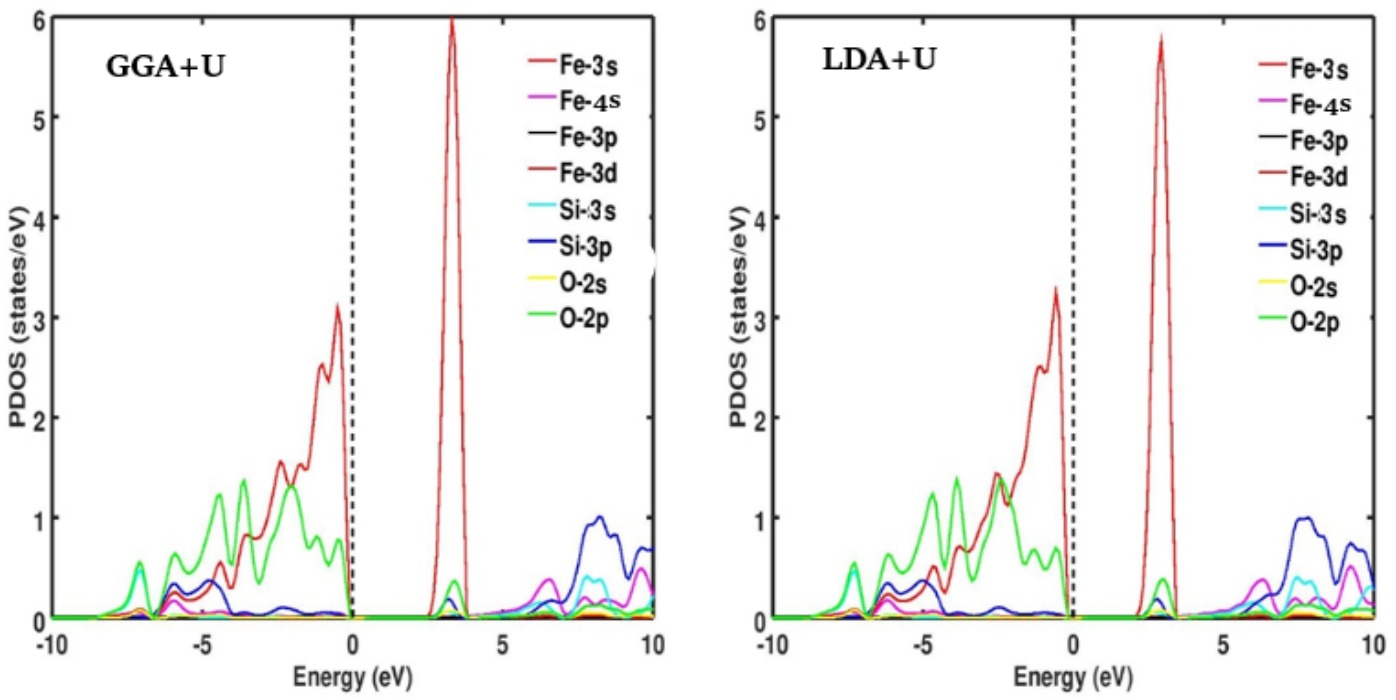

Figure 3. Projected Density of States for $\mathrm{Fe}_{2} \mathrm{SiO}_{4}$ using $\mathrm{GGA}+\mathrm{U}$ and LDA+U

The projected density of states (PDOS) of the bulk $\mathrm{Fe}_{2} \mathrm{SiO}_{4}$ calculated by means of both GGA+U and $\mathrm{LDA}+U$ methods are presented in Figure 3. The bands structure in Fig. 3 are separated by four portions using both the two tecniques. The valence bands (VBs) have two portions. The lowest valence band located around -19.2 to $-16.8 \mathrm{eV}$ arises from the $\mathrm{O} 2 \mathrm{~s}$ state and Si $3 p$ state for $\mathrm{GGA}+U$ while that of $L D A+U$ the two states are situated at $-8.2 \mathrm{eV}$ and $-3.1 \mathrm{eV}$ respectively. The VBs using GGA+U (LDA+U) located from $-8.4 \mathrm{eV}$ and $-3.1 \mathrm{eV}(-8.2 \mathrm{eV}$ and $-3.1 \mathrm{eV})$ mainly consist of the Si $3 p$ state and Fe $4 \mathrm{~s}$ state. The upper ones located between $-2.7 \mathrm{eV}$ and the Fermi level for GGA+U and that of $L D A+U$ from -2.6 eV - Fermi level, originate from the Fe $3 d$ state and $\mathrm{O} 2 p$ state. The CBs (conduction bands) using the GGA+U (LDA+U) are located around 2.7 3.9 eV and $4.8 \mathrm{eV} \sim 10.0 \mathrm{eV}(2.4 \mathrm{eV} \sim 3.6 \mathrm{eV}$ and $4.1 \mathrm{eV} \sim 10.0 \mathrm{eV})$. The CBs positioned at $2.7 \mathrm{eV} \sim 3.9 \mathrm{eV}((2.4 \mathrm{eV} \sim 3.6 \mathrm{eV})$ mainly are contributed by the $\mathrm{Fe} 3 \mathrm{~d}$ state together with the Fe $3 p$ state, and the other ones are mainly contributed by the Fe $4 s$ state and Si 3s state. 


\section{Conclusions}

We have successfully used density functional approach within the GGA and LDA approximations and demonstrated a good description of the structural and electronic properties of $\mathrm{Fe}_{2} \mathrm{SiO}_{4}$ spinel. The GGA and LDA energy bands structure of $\mathrm{Fe}_{2} \mathrm{SiO}_{4}$ fayalite is qualitatively incorrect since this mineral is described as a band metal by GGA and LDA, whereas it is experimentally an insulator with a band gap of $4.22 \mathrm{eV}$. However, when GGA $+U / L D A+U$ was incorporated due to $3 \mathrm{~d}$ electrons of $F e$ atom we found the band gap to be $3.11 \mathrm{eV}\left(\mathrm{GGA}+U\right.$ and $2.88 \mathrm{eV}(\mathrm{LDA}+U)$. Also the bottom most $\mathrm{CB}$ for $\mathrm{Fe}_{2} \mathrm{SiO}_{4}$ spinel is well dispersive, which means that in these materials electrical current can be transported by $\mathrm{CB}$ electrons. In general, the results obtained suggest that, the $\mathrm{Fe}_{2} \mathrm{SiO}_{4}$ spinel is potential candidates for optoelectronic applications

\section{Acknowledgments}

We would like to thank the Department of Physics, Universiti Teknologi Malaysia for the use of its facilities and Tetfund (TETF/ES/ SL.UNIV/KAFIN-HAUSA/ASTD/2017) through Sule Lamido University Kafin-Hausa, Jigawa State, Nigeria for granting fellowship.

\section{References}

[1] R.L. Vekariya, A. Dhar, P.K. Paul, S. Roy, An overview of engineered porous material for energy applications: a mini-review, lonics (Kiel). 24 (2018) 1-17. doi:10.1007/s11581-017-2338-9.

[2] K. Fleischer, E. Norton, D. Mullarkey, D. Caffrey, I. V Shvets, Quantifying the Performance of P-Type Transparent Conducting Oxides by Experimental Methods, Materials (Basel). 10 (2017) 1-14. doi:10.3390/ma10091019.

[3] A.N. Banerjee, K.K. Chattopadhyay, Recent developments in the emerging field of crystalline p-type transparent conducting oxide thin films, Prog. Cryst. Growth Charact. Mater. 50 (2005) 52-105. doi:10.1016/j.pcrysgrow.2005.10.001.

[4] R. Martins, S. Nandy, A.N. Banerjee, E. Fortunato, R. Martins, A Review on Cu2O and Cul-Based p-Type Semiconducting Transparent Oxide Materials: Promising Candidates for New Generation Oxide Based Electronics A Review on $\mathrm{Cu} 2 \mathrm{O}$ and $\mathrm{Cu}$ I -Based p -Type Semiconducting Transparent Oxide Materials: Promising Candidates, (2013). doi:10.1166/rase.2013.1045.

[5] A. Stadler, Transparent Conducting Oxides-An Up-To-Date Overview, Materials (Basel). 5 (2012) 661-683. doi:10.3390/ma5040661.

[6] T.R. Paudel, A. Zakutayev, S. Lany, M. D’Avezac, A. Zunger, Doping rules and doping prototypes in A2BO4spinel oxides, Adv. Funct. Mater. 21 (2011) 4493-4501. doi:10.1002/adfm.201101469.

[7] M.H. Sorour, H.A. Hani, M.M.H. El-sayed, A.A. Mostafa, H.F. Shaalan, Synthesis, Characterization and Performance Evaluation of Lithium Manganese Oxide for Lithium Adsorption, Egypt. J. Chem. 60 (2017) 697710. doi:10.21608/ejchem.2017.4170.

[8] N. Karar, S. Raj, F. Singh, R.E. Kroon, H. Cao, P. Lu, N. Cai, X. Zhang, Z. Yu, T. Gao, S. Wang, H. Lashgari, A. Boochani, A. Shekaari, S. Solaymani, E. Sartipi, R.T. Mendi, V. Dimitrova, J. Tate, Z.A. Tsegaye, Density Functional Theory Studies of Electronic and Optical Properties of ZnS Alloyed with Mn and Cr Zenebe Assefa Tsegaye, Appl. Surf. Sci. 352 (2016) 134-138. doi:10.1016/j.jmmm.2013.10.008.

[9] M. Yousaf, Q. Mahmood, M. Hassan, M. Rashid, A. Laref, Ab Initio Study of Electronic, Magnetic, and Thermoelectric Response of ZTi2O4 ( $Z=M g, Z n$, and Cd) Through mBJ Potential, J. Supercond. Nov. Magn. 31 (2018) 3793-3801. doi:10.1007/s10948-018-4646-3.

[10] L. Azzouz, M. Halit, A. Allal, S. Maabed, M. Bouchenafa, R. Ahmed, T. Seddik, A. Bouhemadou, R. Khenata Structural, electronic, optical and elastic properties of layered rhombohedral compounds ALaSe $2(A=K, R b)$ : Insights from an ab initio study, Int. J. Mod. Phys. B. 33 (2019) 1-22. doi:10.1142/S021797921950084X.

[11] H. Liu, V. Avrutin, N. Izyumskaya, Ü. Özgr, H. Morkoç, Transparent conducting oxides for electrode applications in light emitting and absorbing devices, Superlattices Microstruct. 48 (2010) 458-484. doi:10.1016/j.spmi.2010.08.011.

[12] Q. Williams, E. Knittle, R. Reichlin, S. Martin, R. Jeanloz, Structural and electronic properties of Fe 2 SiO 4 fayalite at ultrahigh pressures: Amorphization and gap closure, J. Geophys. Res. 95 (1990) 21549. doi:10.1029/JB095iB13p21549.

[13] C.Y. Zhang, X. Ben Wang, X.F. Zhao, X.R. Chen, Y. Yu, X.F. Tian, First-principles calculations of structure and elasticity of hydrous fayalite under high pressure, Chinese Phys. B. 26 (2017). doi:10.1088/16741056/26/12/126103.

[14] Z. Wu, A. Mottana, A. Marcelli, C.R. Natoli, E. Paris, Theoretical analysis of X-ray absorption near-edge structure in forsterite, Mg2SiO4-Pbnm, and fayalite, Fe2SiO4-Pbnm, at room temperature and extreme conditions, Phys. Chem. Miner. 23 (1996) 193-204. doi:10.1007/BF00220730.

[15] M. Cococcioni, A. Dal Corso, S. de Gironcoli, Structural, electronic, and magnetic properties of Fe2SiO4 fayalite: Comparison of LDA and GGA results, Phys. Rev. B. 67 (2003) 094106. doi:10.1103/PhysRevB.67.094106. 
[16] S. Stackhouse, L. Stixrude, B.B. Karki, Determination of the high-pressure properties of fayalite from firstprinciples calculations, Earth Planet. Sci. Lett. 289 (2010) 449-456. doi:10.1016/j.epsl.2009.11.033.

[17] M. Belabbas, O. Arbouche, M. Zemouli, Y. Benallou, M. Benchehima, M. Ameri, Ab initio study of novel III-V nitride alloys B1-xTIxN for optoelectronic applications, Comput. Condens. Matter. 16 (2018) e00309. doi:10.1016/j.cocom.2018.e00309.

[18] W. Setyawan, S. Curtarolo, High-throughput electronic band structure calculations: Challenges and tools, Comput. Mater. Sci. 49 (2010) 299-312. doi:10.1016/j.commatsci.2010.05.010.

[19] A. Manzar, G. Murtaza, R. Khenata, S. Muhammad, Hayatullah, Electronic band profile and optical response of spinel Mgln 204 through modified becke - Johnson potential, Chinese Phys. Lett. 30 (2013). doi:10.1088/0256-307X/30/6/067401.

[20] A. Marini, C. Hogan, M. Grüning, D. Varsano, yambo: An ab initio tool for excited state calculations, Comput. Phys. Commun. 180 (2009) 1392-1403. doi:10.1016/j.cpc.2009.02.003.

[21] F.C. Zhang, H.W. Cui, X.X. Ruan, W.H. Zhang, First-principles study of structural and electronic properties of CdO, J. Chem. Pharm. Res. 6 (2014) 1658-1662. doi:10.1016/j.spmi.2014.09.014.

[22] P. Giannozzi, S. Baroni, N. Bonini, M. Calandra, R. Car, C. Cavazzoni, D. Ceresoli, G.L. Chiarotti, M Cococcioni, I. Dabo, A. Dal Corso, S. De Gironcoli, S. Fabris, G. Fratesi, R. Gebauer, U. Gerstmann, C. Gougoussis, A. Kokalj, M. Lazzeri, L. Martin-Samos, N. Marzari, F. Mauri, R. Mazzarello, S. Paolini, A. Pasquarello, L. Paulatto, C. Sbraccia, S. Scandolo, G. Sclauzero, A.P. Seitsonen, A. Smogunov, P. Umari, R.M. Wentzcovitch, QUANTUM ESPRESSO: A modular and open-source software project for quantum simulations of materials, J. Phys. Condens. Matter. 21 (2009) 395502. doi:10.1088/0953-8984/21/39/395502.

[23] J.P. Perdew, K. Burke, M. Ernzerhof, Generalized Gradient Approximation Made Simple, Phys. Rev. Lett. 77 (1996) 3865-3868. doi:10.1103/PhysRevLett.77.3865.

[24] A. Dal Corso, Pseudopotentials periodic table: From H to Pu, Comput. Mater. Sci. 95 (2014) 337-350. doi:10.1016/j.commatsci.2014.07.043.

[25] V.I. Anisimov, J. Zaanen, O.K. Andersen, Band theory and Mott insulators: Hubbard U instead of Stoner I, Phys. Rev. B. 44 (1991) 943-954. doi:10.1103/PhysRevB.44.943.

[26] M.M. Obeid, Y. Mogulkoc, S.J. Edrees, Y.O. Ciftci, M.M. Shukur, M.M.H. Al-Marzooqee, Analysis of the structural, electronic, elastic and thermodynamic properties of CuAl2X4(X = O, S) spinel structure, Mater. Res. Bull. 108 (2018) 255-265. doi:10.1016/j.materresbull.2018.09.013.

[27] M. Stoica, C. S Lo, P-type zinc oxide spinels: application to transparent conductors and spintronics, New J. Phys. 16 (2014) 055011. doi:10.1088/1367-2630/16/5/055011.

[28] H. Sadouki, A. Belkadi, Y. Zaoui, L. Beldi, B. Bouhafs, S. Méçabih, B. Abbar, Ab-initio prediction of halfmetallicity in Lithium chalcogenides compounds $\mathrm{LiX}(\mathrm{X}=\mathrm{S}$, Se and Te) in zinc-blende and wurtzite structures, Comput. Condens. Matter. 16 (2018). doi:10.1016/j.cocom.2018.e00318.

[29] S.Z. Karazhanov, P. Ravindran, Ab Initio Study Of Double Oxides ZnX2O4 (X=Al, Ga, In) Having Spinel Structure, J. Am. Ceram. Soc. 93 (2010) 3335-3341. doi:10.1111/j.1551-2916.2010.03864.x.

[30] S.H.G.; K. Langer, Single crystal spectra of olivines in the range 40,000-5,000 $\mathrm{cm}-1$ at pressures up to 200 kbar, Am. Mineral. 67 (1982) 343-348. 